Volumen $20 n^{\circ} 22017$

\title{
Editorial
}

\section{Externalización del entrenamiento en Diálisis Peritoneal: riesgos sin beneficio}

El objeto de este editorial no es informar al lector de que la gestión clínica, en demasiadas ocasiones, no se basa en la evidencia científica ni en la opinión de los profesionales; si se lograra invertir esta tendencia, gestión y ciencia, avanzarían más y más eficientemente.

El objeto de este editorial es revisar el escenario actual y futuro de la externalización del entrenamiento en diálisis peritoneal (DP).

Han saltado las alarmas debido a que el Servicio de Salud del Gobierno de las Islas Baleares, en su pliego de prescripciones técnicas que rigen el contrato de servicio para la prestación del tratamiento sustitutivo de la función renal ${ }^{2}$, abre, de manera ambigua, una puerta para que las visitas domiciliarias y la formación post-entrenamiento corran a cargo de las empresas adjudicatarias (www.plataformadecontratacion.caib.es).

El personal de la empresa, debidamente titulado y con experiencia demostrada, deberá encargarse de la formación del paciente en su domicilio para: correcto uso de los equipos y material fungible, adiestramiento en los protocolos para la correcta administración de los tratamiento, así como, del seguimiento de los mismos, comprobación del entorno y proponer al paciente medidas de adecuación, realizar las recomendaciones sanitarias pertinentes y motivar al paciente en la realización del tratamiento.

Por supuesto, la formación será coordinada por el centro prescriptor (unidad de DP). Los profesionales sanitarios de la unidad de DP certificarán la capacitación del paciente para inicio en domicilio de la terapia y recaerá sobre ellos, la responsabilidad clínica del paciente.

Es este oxímoron legal (lo dice todo sin decir nada) y esta ambigüedad (no se sabe la categoría profesional, cómo se demuestra la experiencia ni en qué, qué protocolos instruyen, cuáles son las medidas sanitarias pertinentes...) lo que hace saltar las alarmas, obligando a la Sociedad Española de Enfermería Nefrológica a posicionarse.

El entrenamiento en DP es considerado por la comunidad científica como el factor más importante para lograr la excelencia en DP. La International Society of Peritoneal Dialysis (ISPD), en 2011, fundamentaba el éxito de la DP en: "La enfermera de DP (responsable del entrenamiento), la unidad de DP y el paciente".

Anteriormente, en su posicionamiento de 2006², advierte: "Existe una falsa idea generalizada de que cualquier persona puede capacitar a un paciente domiciliario". "El entrenador debe comprender la complejidad del aprendizaje de adultos, aplicada al paciente urémico. El entrenador es un componente central en un programa de DP exitoso".

Además, debemos resaltar la importancia de la experiencia del entrenador en el perfecto devenir del entrenamiento y en la disminución de infecciones peritoneales. Así lo justifica un estudio de cohortes de 305 pacientes, que evidenció que los pacientes entrenados por enfermeras con más de 15 años de experiencia tienen menor riesgo de sufrir peritonitis por gérmenes Gram positivos 3 .

En resumen, la bibliografía muestra que el entrenamiento en DP es primordial, y su responsable debe ser una enfermera con amplia experiencia en el aprendizaje de adultos urémicos ${ }^{4,5}$ (en nuestros hospitales tenemos muchas); por eso, su posible externalización nos parece injustificada. La industria tiene grandes profesionales, muchos de ellos enfermeras y amigas, que son excelentes formadores de profesionales y que nos ayudan en nuestra difícil labor de capacitar pacientes, pero sin experiencia en el entrenamiento.

El entrenamiento no se debe compartimentar y es más que la capacitación anterior al comienzo en domicilio. Se trata de un proceso continuo $0^{6,7}$, se debería iniciar antes de la colocación del catéter y se debería continuar después del inicio del tratamiento, a través de las visitas domiciliarias y el re-entrenamiento. La ISPD recomienda que sea realizado por la misma enfermera.

La presencia de un entrenador a lo largo de todo el proceso de capacitación facilita la continuidad de cuidados y el desarrollo del Apego Peritoneal (Vínculo especial que se establece, durante el entrenamiento, entre el paciente de DP y su entrenador, basada en la Confianza, Comprensión y Comunicación) ${ }^{8,9}$. Apego peritoneal y continuidad de cuidados, son claves, para la excelencia del programa, la satisfacción del paciente y la investigación. 
Por si fuera poco, la ISPD apostilla en sus recomendaciones del 2006: “Los entrenadores deberían asumir la responsabilidad de los errores razonablemente previsibles del paciente, que podrían haberse prevenido mediante la educación adecuada". Si se compartimenta el entrenamiento, se debería compartir la responsabilidad clínica.

En sus 41 años, la SEDEN ha buscado la excelencia en el cuidado del paciente renal y en la formación de la enfermera nefrológica, a través de la evidencia científica. De acuerdo a este principio fundacional, debemos decir que este ambiguo intento de externalización de parte del entrenamiento en DP reporta múltiples riesgos sin beneficio.

Nuestro máximo respeto a los profesionales de la industria farmacéutica, que tanto nos ayudan y nos han hecho crecer en nuestra profesión.

Reiteramos a los responsables de gestión nuestro ofrecimiento de colaboración, otro de nuestros principios fundacionales. Plantéense la gestión clínica basada en la evidencia científica, nos irá mucho mejor a todos, pero, hasta entonces, apliquen el sentido común. Tienen excelentes profesionales con experiencia en el entrenamiento en sus hospitales: escúchenlos, foméntenlos, auméntenlos, cuídenlos... y no busquen fuera lo que tiene en casa. Eso solo nos reportará muchos riesgos sin beneficio.

\section{Miguel Núñez Moral \\ Entrenador de DP y Vocal de Diálisis Peritoneal de la SEDEN}

Agradecido a Ester y Samuel, por sus generosas contribuciones a este editorial

http://dx.doi.org/10.4321/S2254-288420170000300001

\section{Bibliografía}

1. Pliego de prescripciones técnicas que rigen el contrato de servicio para la prestación del tratamiento sustitutivo de la función renal. [Consultado 14 julio 2017] Disponible en: http:// www.plataformadecontractacio.caib.es/Licitacion. jsp? id=42558\&id0rganoContratacion=-1 \&pagina $=\& i d i=e s \& i d T i p o C o n t r a t o=S E R V I C I 0 \& b a j a=N \& i$ dTipoProcedimiento $=\mathrm{CMAJ}$

2. Bernardini J, Price V, Figueiredo A. Pautas/ recomendaciones de la ISPD. Capacitación de pacientes en diálisis peritoneal. Perit Dial Int 2006; 26: 625-632.

3. Yang $\mathrm{Z}, \mathrm{Xu} \mathrm{R}$, Zhuo $\mathrm{M}$ et al. Advanced nursing experience is beneficial for lowering the peritonitis rate in patients on peritoneal dialysis. Perit Dial Int 2012; 32: 60-66.

4. Zhang L, Hawley CM, Johnson DW. Focus on peritoneal dialysis training: working to decrease peritonitis rates. Nephrol Dial Transplant 2016; 31: 214-222.
5. Figueiredo A, Bernardini J, Bowes E, Hiramatsu $M$, Price $V$, Su C et al. ISPD guidelines/recommendations. A syllabus for teaching peritoneal dialysis to patients and caregivers. Perit Dial Int 2016; 36: 592-605

6. Hurst $H$. A new peritoneal dialysis training guideline from the ispd nursing committee. Perit Dial Int 2016;36: 585-586.

7. Luongo M, Prowant B. PD Peritoneal Dialysis Program organization and management. In Khanna R, Krediet RT.Nolph and Gokal's Texbook of PD. Neww York:Springer;2009.335-370.

8. Rebolo A, Pòns ME, Macias MJ, Cabrera S. Valoración de la acogida de pacientes que inician terapia renal sustitutiva: Nivel de satisfacción. Enferm Nefrol 2015;18(2):97-102.

9. Terraza R, Vargas L, Vázquez ML. La coordinación entre niveles asistenciales: una sistematización de sus instrumentos y medidas. Gac Sanit. 2006;20(6):485-95. 